\title{
Audit of Nurse led Skin Prick Testing Clinic: Is it worthwhile?
}

\author{
Miss J Narayan, Mr P Jervis \\ ENT Department Northampton General Hospital NHS Trust, Northampton, UK
}

Background:

Skin Prick Test (SPT) detects presence of allergen specific IgE ${ }^{1}$. Contra-indications include recent anaphylaxis, skin conditions ${ }^{2}$ and certain types of medication ${ }^{3}$; some of which can also interfere with the test results. In vivo testing clinic could be expensive ${ }^{4}$ with adverse effects ${ }^{5}$

\section{Objectives:}

To assess safety of nurse led skin prick testing

\section{Audit Standards:}

$100 \%$ adherence to contra-indications

- No adverse outcomes

- Accurate and complete documentation

- Clinic attendance of $\geq 94 \%$ with no same day cancellations

\section{Methods:}

Retrospective audit of Skin Prick Test clinic run by ENT Nurse Practitioners of $(45+2)$ consecutive patients from Jul 2016 to Feb 2017

Data taken from the ENT outpatients electronic records, clinical notes, allergy questionnaire $\&$ form

- 2 patients did not have the test - aural care \& non ENT patients

\section{Medications that can affect the test: $\&$ for how long ${ }^{6}$}

\section{H1 antihistamines suppress skin reactivity for 1-7 days}

- $1^{\text {st }}$ generation $1-3$ days

- $\quad 2^{\text {nd }}$ generation up to 7 days

- $\quad 3$ destoratidine) (e.g. fexofenadine, levocetirizine \&

- antihistamine nasal sprays $\&$ antihistamine eye drops 3 days $\mathrm{H} 2$ receptor antagonists (eg, ranitidine, cimetidine) - at least 48 hours Antihistamines used for nonallergic diseases, such as phenothiazine antiemetics (e.g. promethazine, prochlorperazine alimemazine) or those for vertigo/mocion sickness or insomnia (e.g. meclizine, doxylamine) may block skin reactivity for up to 2 weeks

If a patient has not withheld antihistamines for the full recommended time, interference can still be present even though the histamine reaction Tricyclic antidepressants -2 weeks

Omalizumab -6 months

Consider RAST: Methotrexate; Beta blockers and ACE inhibitors could interfere with treatment of anaphylaxis

Drugs that don't interfere with SPT: Inhaled, intranasal and oral glucocorticoids, SSRI, Leukotriene receptor antagonists

What we tell our patients

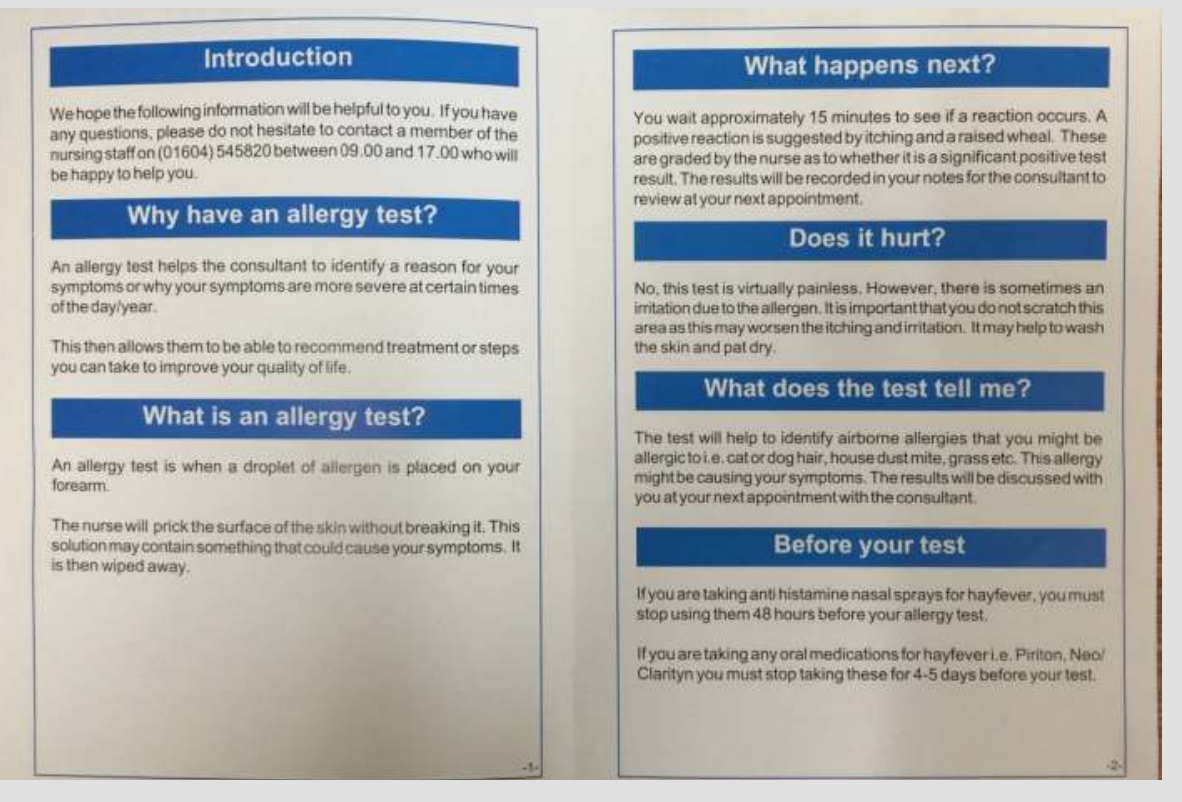

\section{Nurse led SPT Clinic}

Posters in clinic remind clinicians of contra-indications to referring patients for SPT

Patients fill a questionnaire, that confirms their medication, allergies and anaphylaxis history at appointment

Nurse led SPT done in booked clinic and recorded on a form No guidelines available on the Trust intranet

\section{Results 1: Patient Demographics} Age and Sex distribution

$$
\text { Number of }
$$$$
\text { Patients }
$$

Male

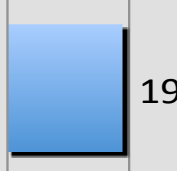

Female

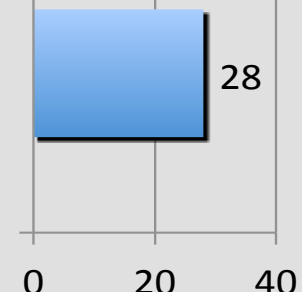

\section{What we tell our doctors}

Skin Prick Testing

When referring patients for skin prick testing please be aware of our Hospital protocol.

Skin tests should not be performed on: Children under the age of 16

Patients who have a history of anaphylaxis Pregnant patients

s taking beta-blockers

Patients taking oral antihistamines

Patients taking systemic steroids

Patients taking tri-cyclic antidepressants

Patients taking monoamine-oxidase inhibitors

Areas of active skin disease

For these patients RAST blood tests can be considered

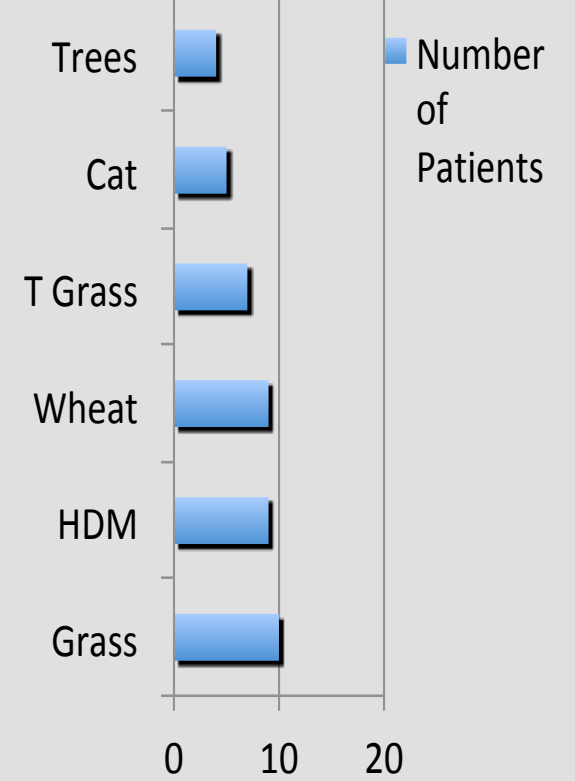

Results 4: Responses to patient Questionnaire \& Test

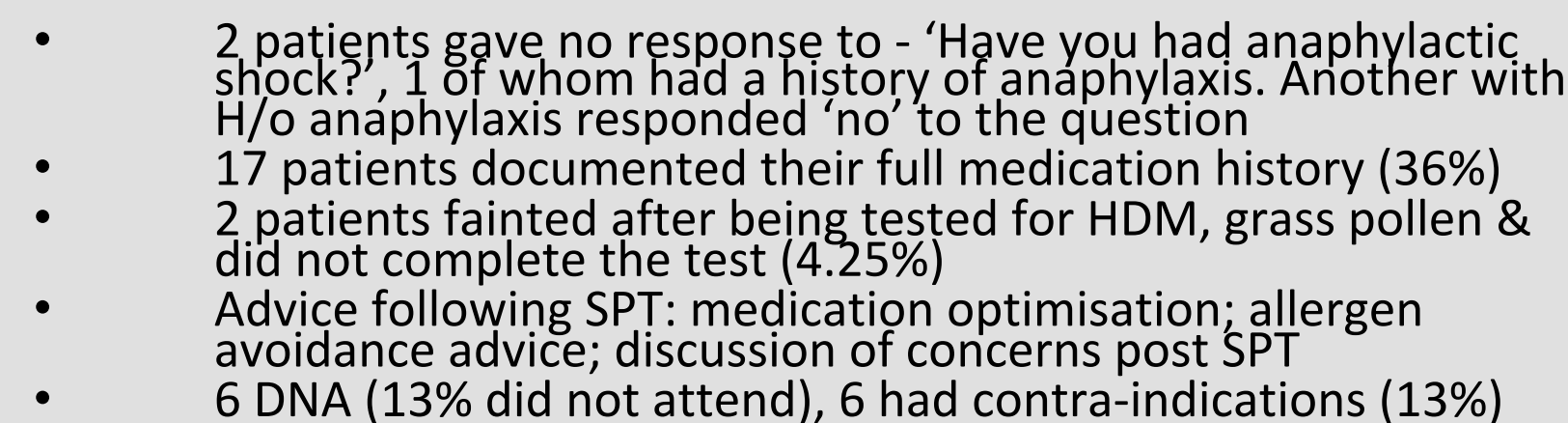

avoidance advice; discussion of concerns post SPT

\section{Summary and Conclusions}

- $86.67 \%$ attendance with further $13.33 \%$ test cancellation on the day. $4.44 \%$ suffered adverse reaction. There was $97 \%$ adherence to contraindications.

- $\quad 13.33 \%$ were referred for SPT by clinicians despite displayed contra-indications of which $2.22 \%$ underwent SPT.

- Patient questionnaires are not accurately completed and can lead to administering the test to patients who should not be tested

- $\quad$ Growing list of drugs interfere with SPT \& compliance with contra-indications is limited by human factors. $87 \%$ attendance falls short of the Trust attendance target and calculations4 show SPT clinic is more expensive than Antigen specific IgE test, with

\section{Suggested changes to the Skin prick testing clinic:}

- Update the Contra-indications

- Better compliance with documentation

- Focused history taking of medication \& allergies before referral

- Patients should bring their Medication list

- Consider making the protocol available on the Trust intranet potential for adverse events.

\section{References}

\author{
1.H Nolte, K Kowal, L DuBuske, Overview of skin testing for allergic disease UpToDate www.uptodate.com \\ (C)2016 UpToDate ${ }^{\circledR}$ \\ 2, 5. BSACI Nurses in Allergy Committee. Adult Skin Prick Testing. Available \\ from: http://www.bsaci.org/LiteratureRetrieve.aspx?ID=141819. Accessed on 05 February 2018 \\ 3. Lucie H, Adriano M, Karl-Christian B, Megon B, Guido B, Ulf D, Stephen D, Wytske F, Mark G, Tari H, \\ Ana T B, Stefan W, Howard M and Richard L. The skin prick test - European standards.Clin TransI \\ Allergy. 2013; 3: 3. Available from: doi: 10.1186/2045-7022-3-3 \\ 4. National Institute for Health and Care Excellence (NICE) Available \\ from: https://www.nice.org.uk/guidance/cg116/evidence/full-guideline-appendix-3-healtheconomics- \\ pdf-136470064 pg 17 Accessed on 05 February 2018 \\ 6. Table 2: Potential interference of medications with the skin test reaction (adapted from Demoly (2003) [23]; \\ Rueff (2010) [24] and Position Paper: Allergen standardization and skin tests: The European Academy of Allergy
}

